\title{
Procesamiento de una bebida nutritiva a base de alfalfa (Medicago sativa) y maracuyá (Passiflora edulis) "Alfamar"
}

\author{
Processing of a nutritive drink based on alfalfa (Medicago sativa) and passion fruit \\ (Passiflora edulis) "alfamar"
}

Nicodemo C. Jamanca Gonzáles ${ }^{1}$, Ronald F. Rodríguez Espinoza', Nilthon Santa María Podestá ${ }^{2}$

\section{RESUMEN}

Objetivo: Elaborar una bebida nutritiva a partir de extracto de alfalfa (Medicago sativa) y zumo de maracuyá (Passiflora edulis) para determinar su mezcla óptima. Materiales y Métodos: El diseño experimental utilizado fue un DCCR (Diseño compuesto central rotacional) o factorial $2^{2}$ con 11 experimentos, considerando los niveles de zumo de maracuyá y extracto de alfalfa como variables independientes. Los efectos de estas variables fueron evaluados en las propiedades fisicoquímicas y sensoriales con 20 panelistas no entrenados. Los resultados fueron analizados a través de la metodología de superficies de respuesta utilizando el software Statistica-Statsoft v. 8.0. Resultados: Los resultados muestran que el extracto de alfalfa no tiene influencia significativa en las propiedades fisicoquímicas estudiadas; y el zumo de maracuyá tiene influencia significativa en las variables de $\mathrm{pH}$, acidez y porcentaje de sólidos solubles; al aumentar las concentraciones de zumo de maracuyá los valores de acidez y porcentaje de sólidos solubles de la pulpa diluida aumentan; y por el contrario al disminuir la concentración, el pH final aumenta. Conclusiones: La alfalfa tiene una influencia significativa en las características sensoriales como sabor, consistencia y apariencia; mientras que el color del producto es influenciado por la interacción del zumo de maracuyá y de alfalfa. El mejor tratamiento es la mezcla de $25,6 \%$ de zumo de maracuyá y $2,9 \%$ de extracto de alfalfa, que presenta un $\mathrm{pH}$ de 2,5; acidez de 0,99\% y $12^{\circ}$ Brix; mostrando además una alta probabilidad de intención de compra.

Palabras clave: Medicago sativa, Passiflora edulis, diseño compuesto central rotacional, bebida nutritiva.

\section{ABSTRACT}

Objective: Make a nutritious beverage from alfalfa (Medicago sativa) extract and juice of passion fruit (Passiflora edulis) to determine your optimal mix. Material and Methods: The experimental design used was a (rotational central composite design) DCCR or factorial 22 with 11 experiments, considering the levels of passion fruit juice and extract from alfalfa as independent variables. The effects of these variables were evaluated in the physicochemical and sensorial properties with no trained panelists 20 . The results were analyzed through the methodology of response surfaces using Statistica-Statsoft software v. 8.0. Results: The results show that alfalfa extract does not have significant influence in the studied physicochemical properties; and passion fruit juice has significant influence on the variables $\mathrm{pH}$, acidity and percentage of soluble solids; to increase the concentrations of passion fruit juice acidity and percentage of soluble solids of the diluted pulp values increase; and onthe other hand to reduce the concentration, the final $\mathrm{pH}$ increases. Conclusions: Alfalfa has a significant influence onthe sensory taste, consistency and appearance characteristics; While the color of the product is influenced by the interaction of the juice of passion fruit and alfalfa. The best treatment is the mixture of $25.6 \%$ of juice of passion fruit and $2.9 \%$ extract of alfalfa, presenting a $\mathrm{pH}$ of 2.5 ; acidity of $0.99 \%$ and $12^{\circ}$ Brix; showing also a high probability of purchase intent.

Keywords: Medicago sativa, Passiflora edulis, rotational central composite design, nutritious drink.

${ }^{1}$ Facultad de Ingeniería, Universidad Nacional de Barranca. Barranca, Perú

2 IESTP Huarmey. Huarmey, Perú. 


\section{INTRODUCCIÓN}

En la actualidad existe una demanda sobre productos naturales, en ese sentido hay un creciente consumo de productos de origen vegetal principalmente de frutas y hortalizas, por su aporte considerable en vitaminas $y$ minerales.

En esa óptica en el presente trabajo se plantea procesar un producto innovador a partir de la mezcla de extractos de alfalfa y maracuyá, con el fin de obtener una bebida nutritiva y que esté al alcance del consumidor como un alimento nutritivo y de gran aporte para la alimentación.

El producto se justifica porque actualmente existe una gran tendencia hacia el consumo de bebidas energizantes, rehidratantes y refrescantes.

La alfalfa representa uno de los mayores recursos vegetales que no se han explotado adecuadamente en nuestro país en el ámbito alimentario de manera industrial; solo existe un aprovechamiento muy limitado a través de vendedores ambulantes de una bebida muy típica llamada "Emoliente".

Huarmey, cuenta con un ambiente muy apropiado para la producción de la alfalfa y maracuyá, que son respaldados con las estadísticas de producción, que ubican a la alfalfa con el tercer cultivo con mayor extensión de superficies cultivadas; asimismo el maracuyá muestra incrementos muy apreciados en superficies cultivadas a nivel del Valle de Huarmey tal como lo afirma Jamanca (2013).

Estas consideraciones nos permiten establecer como objetivo procesar un producto innovador en la línea de bebidas refrescantes y energéticas para obtener una bebida nutritiva a partir de la alfalfa y maracuyá, incrementando su valor nutricional principalmente en fibras, vitaminas y minerales como lo menciona Reyes, Gómez, Espinoza, Bravo, y Ganoza (2009), de calidad aceptable para el consumidor de todo estrato social y pueda ser ingerido en cualquier momento del día y temporada del año.

\section{MATERIAL Y MÉTODOS}

\section{Materias primas e insumos}

Las materias primas empleadas en el presente trabajo fueron adquiridas en el mercado modelo de la ciudad de Huarmey, procedente del valle del mismo nombre, que se caracteriza por una considerable producción de estos cultivos.

- Alfalfa. La alfalfa pertenece a la familia de las leguminosas. Se trata de una planta perenne, vivaz y de porte erecto. Se trata de un cultivo muy extendido en los países de clima templado. La importancia del cultivo de la alfalfa va desde su interés como fuente natural de proteínas, fibra, vitaminas como retinol y minerales como el hierro, como lo señala Reyes et al, (2009).

- Maracuyá. Es un fruto ovalado y contiene muchas semillas de color negro, envueltas en una sustancia jugosa de sabor agradable. La composición de la fruta de maracuyá es de 50 - $60 \%$ de cáscara, de 30 $40 \%$ de jugo y de 10 a $15 \%$ de semilla. Según Reyes et al, (2009), el fruto se caracteriza por ser rico en hidratos de carbono, ácidos orgánicos y vitamina $A$, aportando en sólo $100 \mathrm{~g}$ de porción $46 \mathrm{cal}$.

- Sacarosa conocida comúnmente como azúcar de caña.

\section{Proceso tecnológico}

En otros países como México existen estudios de aprovechamiento de la alfalfa en la elaboración de bebidas funcionales acompañadas de frutas para mejorar sus atributos sensoriales como lo señala Gonzales, Jiménez, Pérez, Rangel, y Serrano (2003). El proceso tecnológico empleado en el procesamiento de esta bebida nutritiva se muestra en la Figura 1. 


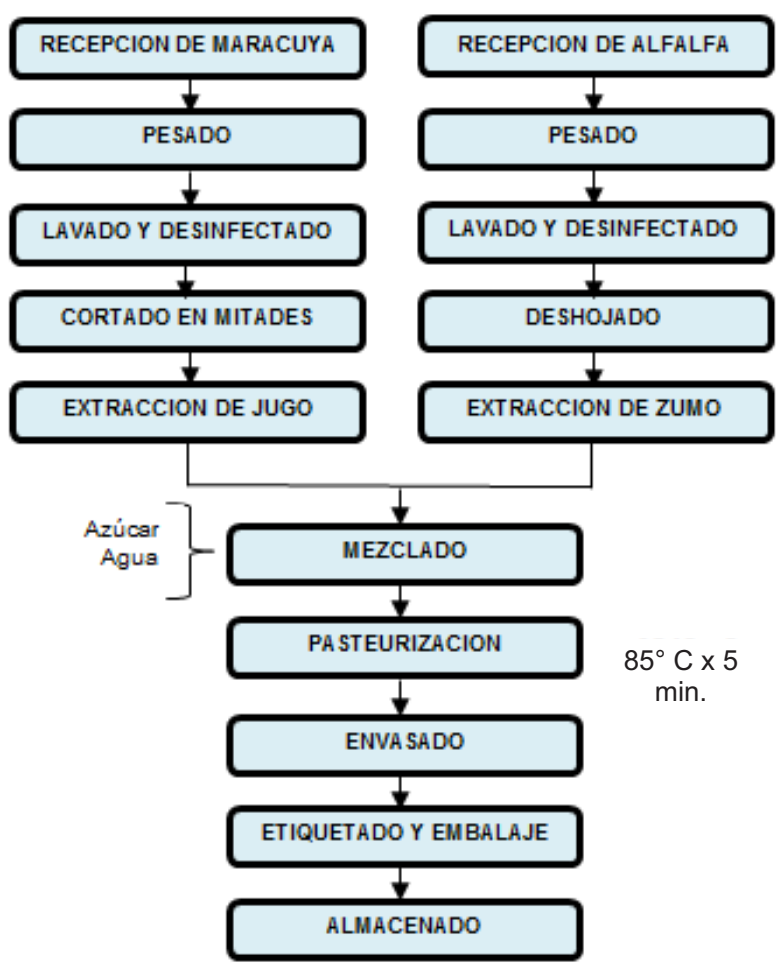

Figura 1. Diagrama de flujo del procesamiento de Alfamar

a) Recepción. Las materias primas se transportan a la sala de procesamiento, evitando su deterioro posterior.

b) Pesado. Es importante realizar el pesado porque permitirá determinar el rendimiento del proceso de producción. Asimismo, nos servirá para determinar las pérdidas en cada operación.

c) Lavado y desinfección. La alfalfa es transportada hasta una mesa de trabajo donde fue seleccionada. Posteriormente se depositó en una tina de lavado para retirar las materias extrañas en general, este lavado dura de 5 a 10 min. Posteriormente se desinfectó con hipoclorito de sodio con una concentración de $5 \mathrm{ml} / /$ durante $5 \mathrm{~min}$. Se deja escurrir antes de entrar a la extracción.

d) Extracción. La alfalfa se deposita en la licuadora donde se realizó la separación de la fibra y el jugo de la misma. La molienda se realiza con la adición de agua en una proporción de 1:3. La distribución de los componentes de la alfalfa es $67 \%$ de hoja y $33 \%$ de tallo.

Para el estudio solo se utilizó las hojas, considerando que son las más jugosas en comparación al tallo que es más duro y reseco.

e) Filtración. La filtración consistió en eliminar aquellas partículas de gran tamaño provenientes de la extracción. Para ello se empleó tamices o coladores.

f) Mezclado. Consistió en el mezclado de todos los insumos: jugo de alfalfa, maracuyá y azúcar. Esta operación se realizó en un recipiente de acero inoxidable. El producto final contiene un $12^{\circ}$ Brix.

g) Pasteurización. La pasteurización se lleva a cabo a una temperatura de $85^{\circ} \mathrm{C}$ por 5 min. Terminado el proceso de pasteurización se llevan a una tina de enfriamiento.

h) Envasado. La bebida fue envasada en botellas de vidrio de $300 \mathrm{ml}$ con tapa rosca, para garantizar la conservación del producto.

i) Etiquetado y embalaje. Se realizó en forma manual con etiquetas previamente diseñadas.

j) Almacenamiento. Una vez empacado el producto se trasladó al área de almacenamiento hasta el día de su distribución.

\section{Diseño experimental}

El diseño experimental utilizado fue un DCCR (Diseño compuesto central rotacional) o factorial $2^{2}$ con 11 experimentos ( 4 experimentos factoriales, 4 experimentos axiales y 3 puntos centrales), considerando los niveles de zumo de maracuyá y extracto de alfalfa como variables independientes, como lo recomienda Rodríguez y Lemma (2005).

Los efectos de estas variables fueron evaluados considerando las propiedades fisicoquímicas $(\mathrm{pH}, \%$ de sólidos solubles final, $\%$ de sólidos solubles de pulpas diluidas y acidez) y propiedades sensoriales evaluadas con 20 panelistas no entrenados.

\section{Análisis estadístico}

Los resultados fueron analizados a través de la metodología de superficies de respuesta utilizando el paquete Statistica - Statsoft v. 8.0. 
Tabla 1. Variables codificadas y reales del diseño experimental

\begin{tabular}{|c|c|c|c|c|}
\hline \multirow[b]{2}{*}{ Ensayos } & \multicolumn{2}{|c|}{ Variables codificadas } & \multicolumn{2}{|c|}{ Variables reales } \\
\hline & $\begin{array}{l}\text { Zumo de } \\
\text { maracuyá }\end{array}$ & $\begin{array}{c}\text { Extracto } \\
\text { de } \\
\text { alfalfa }\end{array}$ & $\begin{array}{l}\text { Zumo de } \\
\text { maracuyá }\end{array}$ & $\begin{array}{c}\text { Extracto } \\
\text { de } \\
\text { alfalfa }\end{array}$ \\
\hline 1 & -1 & -1 & 4,4 & 2,9 \\
\hline 2 & +1 & -1 & 25,6 & 2,9 \\
\hline 3 & -1 & +1 & 4,4 & 17,1 \\
\hline 4 & +1 & +1 & 25,6 & 17,1 \\
\hline 5 & $-1,41$ & 0 & 0,0 & 10,0 \\
\hline 6 & $+1,41$ & 0 & 30,0 & 10,0 \\
\hline 7 & 0 & $-1,41$ & 15,0 & 0,0 \\
\hline 8 & 0 & $+1,41$ & 15,0 & 20,0 \\
\hline 9 & 0 & 0 & 15,0 & 10,0 \\
\hline 10 & 0 & 0 & 15,0 & 10,0 \\
\hline 11 & 0 & 0 & 15,0 & 10,0 \\
\hline
\end{tabular}

\section{RESULTADOS}

Caracterización de la materia prima.

Los resultados de la caracterización física son mostrados en la Tabla 2.

Tabla 2. Características físicas del maracuyá y alfalfa

\begin{tabular}{l|c|c}
\hline Características & $\begin{array}{c}\text { Extracto de } \\
\text { alfalfa }\end{array}$ & $\begin{array}{c}\text { Zumo de } \\
\text { maracuyá }\end{array}$ \\
\hline $\mathrm{pH}$ & 7,0 & 2,5 \\
Acidez $(\%)^{(i)}$ & 0,2 & 1,2 \\
\hline Sólidos solubles & 0,2 & 4,0 \\
\hline
\end{tabular}

$\left(^{*}\right)$ Expresado en $\%$ de ácido cítrico

Evaluación de las características físico químicas de los ensayos

Tabla 3. Características físico químicas de los ensayos

\begin{tabular}{|c|c|c|c|c|c|}
\hline \multirow[t]{2}{*}{$\begin{array}{l}\text { Ensa } \\
\text {-yos }\end{array}$} & \multicolumn{2}{|c|}{$\begin{array}{c}\text { Variables } \\
\text { Independientes }\end{array}$} & \multicolumn{3}{|c|}{ Variables Dependientes } \\
\hline & $\begin{array}{c}\text { Zumo de } \\
\text { maracuyá } \\
(\%)\end{array}$ & $\begin{array}{c}\text { Extracto } \\
\text { de } \\
\text { alfalfa } \\
(\%)\end{array}$ & $\begin{array}{l}\text { \%Sólido } \\
\text { S } \\
\text { solubles } \\
\left({ }^{\circ} \text { Brix) }\right.\end{array}$ & $\mathrm{pH}$ & $\begin{array}{l}\text { Acide } \\
\text { z }(\%)\end{array}$ \\
\hline 1 & 4,4 & 2,9 & 1,0 & 4,0 & 0,18 \\
\hline 2 & 25,6 & 2,9 & 3,5 & 2,5 & 0,99 \\
\hline 3 & 4,4 & 17,1 & 1,0 & 4,0 & 0,20 \\
\hline 4 & 25,6 & 17,1 & 3,5 & 2,5 & 1,06 \\
\hline 5 & 0,0 & 10,0 & 0,5 & 7,0 & 0,03 \\
\hline 6 & 30,0 & 10,0 & 4,5 & 2,5 & 1,00 \\
\hline 7 & 15,0 & 0,0 & 2,0 & 2,5 & 0,61 \\
\hline 8 & 15,0 & 20,0 & 2,5 & 2,5 & 0,68 \\
\hline 9 & 15,0 & 10,0 & 2,0 & 2,5 & 0,67 \\
\hline 10 & 15,0 & 10,0 & 2,0 & 2,5 & 0,68 \\
\hline 11 & 15,0 & 10,0 & 2,0 & 2,5 & 0,69 \\
\hline
\end{tabular}

Tabla 4. Análisis de varianza para el \% sólidos solubles en las mezclas

\begin{tabular}{cccccc}
\hline $\begin{array}{c}\text { Fuente } \\
\text { de } \\
\text { variación }\end{array}$ & $\begin{array}{c}\text { Suma de } \\
\text { cuadrados }\end{array}$ & $\begin{array}{c}\text { Grados } \\
\text { de } \\
\text { libertad }\end{array}$ & $\begin{array}{c}\text { Cuadrados } \\
\text { medios }\end{array}$ & $\begin{array}{c}F \\
\text { calculado }\end{array}$ & $\begin{array}{c}\mathbf{F} \\
\text { tabulado } \\
\mathbf{( 2 , 8 ;} \\
\mathbf{0}, \mathbf{0 5})\end{array}$ \\
\hline $\begin{array}{c}\text { Regresión } \\
\text { Residuos }\end{array}$ & 14,42 & 2 & 7,21 & 240,33 & 4,46 \\
Total & 0,26 & 8 & 0,03 & & \\
\hline
\end{tabular}

Tabla 5. Coeficientes de regresión para el \% sólidos solubles en las mezclas

\begin{tabular}{lcccc}
\hline & $\begin{array}{c}\text { Coeficientes } \\
\text { de } \\
\text { regresión }\end{array}$ & $\begin{array}{c}\text { Error } \\
\text { estándar }\end{array}$ & $\begin{array}{c}\boldsymbol{t}(\mathbf{8}) \\
\begin{array}{c}p- \\
\text { valor* }\end{array}\end{array}$ \\
\hline Media & 2,50 & 0,34 & 7,44 & 0,0007 \\
$\boldsymbol{X}_{\mathbf{1}}(\mathbf{L})$ & $-1,17$ & 0,21 & - & 0,0023 \\
$\boldsymbol{X}_{\mathbf{1}}(\mathbf{Q})$ & 1,03 & 0,25 & 5,68 & 0,0084 \\
$\boldsymbol{X}_{\mathbf{2}}(\mathbf{L})$ & 0,00 & 0,21 & 4,21 & 1,0000 \\
$\boldsymbol{X}_{\mathbf{2}}(\mathbf{Q})$ & $-0,09$ & 0,25 & 0,00 & 0,7178 \\
$\boldsymbol{X}_{\mathbf{1}} \mathbf{X}_{\mathbf{2}}$ & 0,00 & 0,29 & - & 1,0000 \\
& & & 0,38 & \\
& & & 0,00 & \\
\hline
\end{tabular}

X1=Zumo de maracuyá, $\mathrm{x} 2=$ Extracto de alfalfa, L=término lineal $\mathrm{Q}=$ término cuadrático; * Valores estadísticamente significativos al $5 \%$ de significancia $(p<0,05), R 2=98,99$

\section{Siendo la ecuación cuadrática}

$\%$ Sólidos solubles $=2,00+1,33 x_{1}+0,22 x_{1^{2}}$

Donde $\mathrm{x}_{1}=$ zumo de maracuyá $(\%)$.
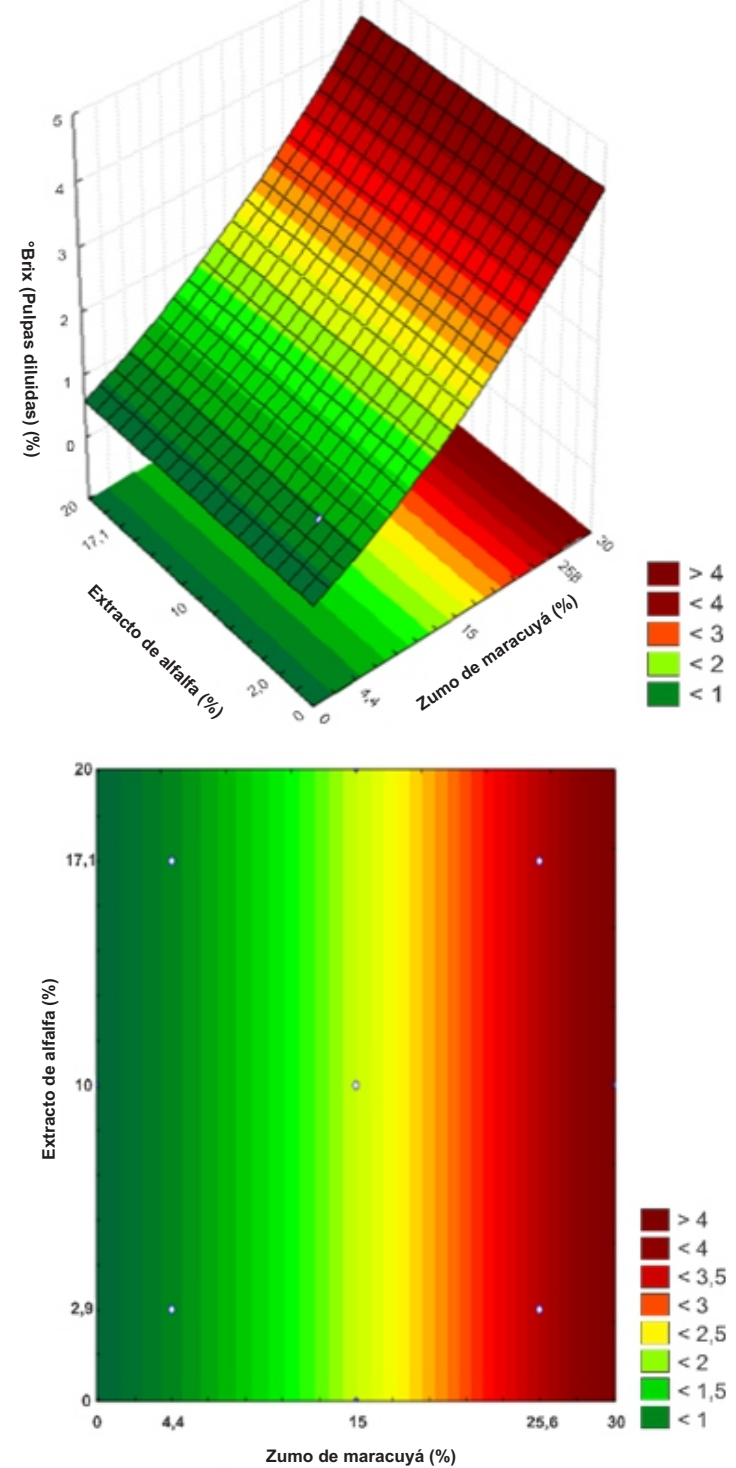

Figura 2. Superficie de respuesta y gráfico de contorno para el porcentaje de sólidos solubles de las pulpas diluidas de los ensayos en función del zumo de maracuyá (\%) y extracto de alfalfa (\%) 
Tabla 6. Coeficientes de regresión para el pH de las mezclas

\begin{tabular}{ccccc}
\hline & $\begin{array}{c}\text { Coeficientes } \\
\text { de regresión }\end{array}$ & $\begin{array}{c}\text { Error } \\
\text { estándar }\end{array}$ & $\boldsymbol{t}(\mathbf{8})$ & p-valor* \\
\hline Media & 2,00 & 0,09 & 20,16 & $<0,0001$ \\
$\boldsymbol{X}_{1}(\mathbf{L})$ & 1,33 & 0,06 & 21,92 & $<0,0001$ \\
$\boldsymbol{X}_{1}(\mathbf{Q})$ & 0,22 & 0,07 & 3,02 & 0,0293 \\
$\boldsymbol{X}_{2}(\mathbf{L})$ & 0,09 & 0,06 & 1,45 & 0,2055 \\
$\boldsymbol{X}_{2}(\mathbf{Q})$ & 0,09 & 0,07 & 1,29 & 0,2515 \\
$\boldsymbol{X}_{1} \boldsymbol{X} \mathbf{X}_{2}$ & 0,00 & 0,09 & 0,00 & 1,0000 \\
\hline
\end{tabular}

$X_{1}=$ zumo de maracuyá, $X_{2}=$ extracto de alfalfa, $L=$ término lineal, $Q=$ término cuadrático. * Valores estadísticamente significativos al $5 \%$ de significancia $(p<0,05) \cdot R^{2}=91,36$.

Siendo la ecuación cuadrática:

$p H=2,50-1,17 x_{1}+1,03 x_{1}^{2}$

Donde $x_{1}=$ zumo de maracuyá (\%).

Tabla 7. Análisis de varianza para el pH en las mezclas

\begin{tabular}{cccccc}
\hline $\begin{array}{c}\text { Fuente } \\
\text { de } \\
\text { variación }\end{array}$ & $\begin{array}{c}\text { Suma de } \\
\text { cuadrados }\end{array}$ & $\begin{array}{c}\text { Grados } \\
\text { de } \\
\text { libertad }\end{array}$ & $\begin{array}{c}\text { Cuadrados } \\
\text { medios }\end{array}$ & $\begin{array}{c}F \\
\text { calculado }\end{array}$ & $\begin{array}{c}F \\
\text { tabulado } \\
\mathbf{( 2 , 8 ;} \\
\mathbf{0 , 0 5}\end{array}$ \\
\hline Regresión & 1,18 & 2 & 0,59 & 196,67 & 4,46 \\
Residuos & 0,02 & 8 & 0,003 & & \\
Total & 1,20 & 10 & 0,12 & & \\
\hline
\end{tabular}

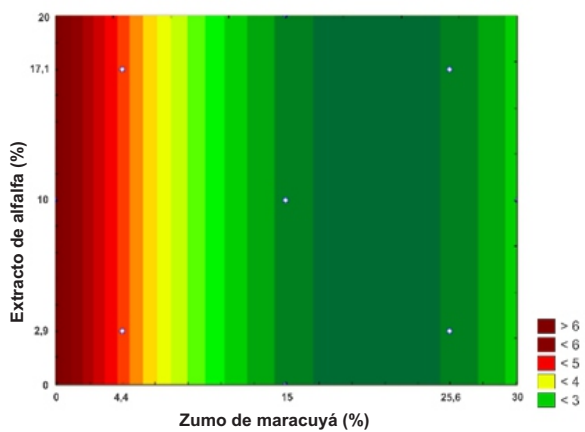

Figura 3. Gráfico de contorno para el pH de las pulpas diluidas de los ensayos en función del zumo de maracuyá (\%) y extracto de alfalfa (\%).

Tabla 8. Coeficientes de regresión para el porcentaje acidez en las mezclas

\begin{tabular}{lcccc}
\hline & $\begin{array}{c}\text { Coeficientes } \\
\text { de } \\
\text { regresión }\end{array}$ & $\begin{array}{c}\text { Error } \\
\text { estándar }\end{array}$ & $\boldsymbol{t}(\mathbf{8})$ & p-valor* \\
\hline Media & 0,68 & 0,03 & 24,07 & $<0,0001$ \\
$\boldsymbol{X}_{1}(\mathbf{L})$ & 0,38 & 0,02 & 21,87 & $<0,0001$ \\
$\boldsymbol{X}_{1}(\mathbf{Q})$ & $-0,08$ & 0,02 & $-3,64$ & 0,0148 \\
$\boldsymbol{X}_{\mathbf{2}}(\mathbf{L})$ & 0,02 & 0,02 & 1,36 & 0,2316 \\
$\boldsymbol{X}_{2}(\mathbf{Q})$ & $-0,01$ & 0,02 & $-0,63$ & 0,5591 \\
$\boldsymbol{X}_{\mathbf{1}} \mathbf{X}_{2}$ & 0,01 & 0,02 & 0,39 & 0,7121 \\
\hline
\end{tabular}

X1=zumo de maracuyá, x2=extracto de alfalfa, L=término lineal, $\mathrm{Q}=$ término cuadrático. * Valores estadísticamente significativos al $5 \%$ de significancia $(p<0,05)$. R2=98,99

Siendo la ecuación cuadrática:

$\%$ Acidez $=0,68+0,38 x_{1}-0,08 x_{12}$

Tabla 9. Análisis de varianza para el \% de acidez en las mezclas

\begin{tabular}{llllll}
\hline $\begin{array}{l}\text { Fuente } \\
\text { de } \\
\text { variación }\end{array}$ & $\begin{array}{l}\text { Sumade } \\
\text { cuadrados }\end{array}$ & $\begin{array}{l}\text { Grados } \\
\text { de } \\
\text { libertad }\end{array}$ & $\begin{array}{l}\text { Cuadrados } \\
\text { medios }\end{array}$ & $\begin{array}{l}\text { F } \\
\text { calculado }\end{array}$ & $\begin{array}{l}\mathrm{F} \\
\text { tabulado } \\
(\mathbf{2 , 8} ; \\
\mathbf{0 , 0 5}\end{array}$ \\
\hline $\begin{array}{l}\text { Regresión } \\
\text { Residuos }\end{array}$ & 17,89 & 2 & 8,95 & 40,68 & 4,46 \\
Total & 19,64 & 8 & 0,22 & & \\
\hline
\end{tabular}
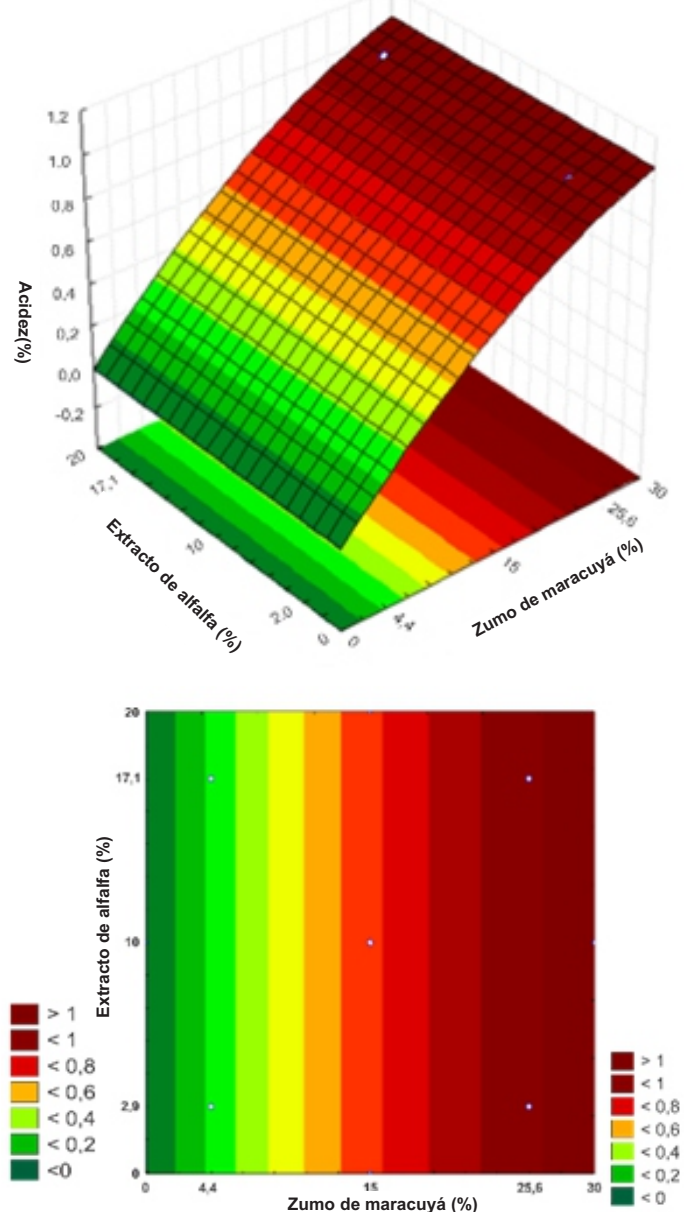

Figura 4. Superficie respuesta y gráfico de contorno para la acidez de las pulpas diluidas de los ensayos en función del zumo de maracuyá (\%) y extracto de alfalfa (\%).

Tabla 10. Coeficientes de regresión para el color de las mezclas

\begin{tabular}{lllll}
\hline & $\begin{array}{l}\text { Coeficientes } \\
\text { de regresión }\end{array}$ & $\begin{array}{l}\text { Error } \\
\text { estándar }\end{array}$ & $\mathbf{t ( 5 )}$ & p-valor* \\
\hline Media & 5,98 & 0,06 & 99,31 & $<0,0001$ \\
$\mathbf{X}_{1}$ (L) & $-0,19$ & 0,07 & $-2,52$ & 0,0529 \\
$\mathbf{X}_{1}$ (Q) & $-0,59$ & 0,09 & $-6,64$ & 0,0012 \\
$\mathbf{X}_{\mathbf{2}}$ (L) & $-0,84$ & 0,07 & - & $<0,0001$ \\
$\mathbf{X}_{\mathbf{2}}(\mathbf{Q})$ & 0,69 & 0,09 & 11,34 & 0,0005 \\
$\mathbf{X}_{\mathbf{1}} \mathbf{X} \mathbf{X}_{\mathbf{2}}$ & 0,03 & 0,10 & 7,88 & 0,8202 \\
\hline
\end{tabular}

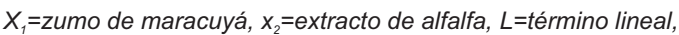
$Q=$ término cuadrático. * Valores estadísticamente significativos al $5 \%$ de significancia $(p<0.05) . R^{2}=98,28$.

Siendo la ecuación cuadrática:

Color $=5,98-0,29 x_{1^{2}}-0,42 x_{2}+0,35 x_{2^{2}}$

Donde $\mathrm{x}_{1}=$ zumo de maracuyá (\%).

Tabla 11. Análisis de varianza para el color de las mezclas

\begin{tabular}{llllll}
\hline $\begin{array}{l}\text { Fuente } \\
\text { de } \\
\text { variación }\end{array}$ & $\begin{array}{l}\text { Suma de } \\
\text { cuadrados }\end{array}$ & $\begin{array}{l}\text { Grados } \\
\text { de } \\
\text { libertad }\end{array}$ & $\begin{array}{l}\text { Cuadrados } \\
\text { medios }\end{array}$ & $\begin{array}{l}\text { F } \\
\text { calculado }\end{array}$ & $\begin{array}{l}\mathrm{F} \\
\text { tabulado } \\
(\mathbf{2 , 8 ;} \\
\mathbf{0 , 0 5}\end{array}$ \\
\hline $\begin{array}{l}\text { Regresión } \\
\text { Residuos }\end{array}$ & 3,04 & 3 & 1,01 & 50,5 & 0 \\
Total & 0,12 & 7 & 0,02 & & \\
\hline
\end{tabular}




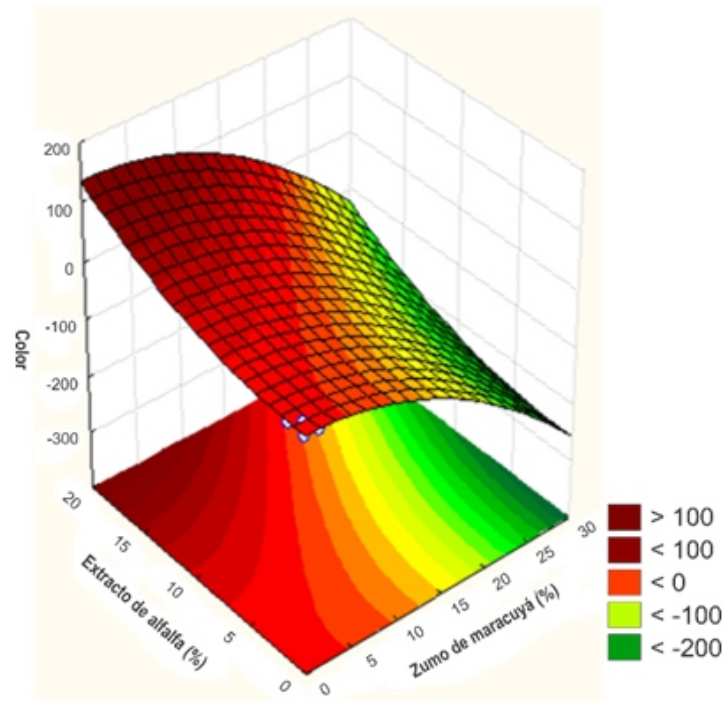

Figura 5. Superficie respuesta y gráfico de contorno para el color de las pulpas diluidas de los ensayos en función del zumo de maracuyá (\%) y extracto de alfalfa (\%).

\section{DISCUSIÓN}

Los resultados del análisis físico químico de la alfalfa y del maracuyá concuerdan con lo establecido por Gonzales (2003) y Franco, Sulca, Delgado, Cáceres y Auccasi (2003).

Los resultados mostrados en las Tablas 4 y 5 y la Figura 2, muestran la influencia significativa $(p<0,05)$ directa del zumo de maracuyá en porcentaje de sólidos solubles de las mezclas, mientras que el extracto de alfalfa no muestra influencia significativa en esta variable.

Las frutas de manera natural contienen azúcares presentes como sacarosa y fructuosa que son medidas a través del contenido del porcentaje de sólidos solubles expresadas en ${ }^{\circ}$ Brix. Asimismo, presentan cantidades importantes de ácidos orgánicos como ácido cítrico, ascórbico y málico que se ven reflejados en su pH, como lo señala Rodríguez (2005). Por ello fue necesario evaluar la influencia de las variables independientes de \% de zumo de maracuyá y extracto de alfalfa en las variables independientes de \% sólidos solubles, $\mathrm{pH}$ y acidez en los diferentes ensayos, cuyos resultados se muestran en la Tabla 3 .

A partir de los resultados mostrados en la Tabla 6,7 y Figura 3 se establece que el extracto de alfalfa no tiene influencia significativa en el pH, mientras que el zumo de maracuyá es la que influye directamente sobre el $\mathrm{pH}$, deduciéndose que a menores valores de zumo de maracuyá el $\mathrm{pH}$ aumenta.
Los resultados mostrados en las Tablas 8,9 y Figura 4, evidencian que el extracto de alfalfa no tiene influencia significativa en la acidez, dependiendo únicamente esta variable de la acidez del maracuyá, deduciéndose que a mayores valores de zumo de maracuyá la acidez aumenta.

\section{Evaluación de las características sensoriales}

La evaluación de las características sensoriales es importante para conocer la aceptabilidad de los diferentes ensayos, para ello se efectuó la prueba sensorial de aceptación de 9 puntos; siendo 9 la máxima puntuación (me gusta muchísimo) y 1 la mínima (me disgusta muchísimo).

Color: El zumo de maracuyá y el extracto de alfalfa tienen influencia significativa en el color del producto, siendo los ensayos son zumo de maracuyá (al $5 \%, 7 \%$ y $15 \%$ ) los que alcanzan mejor valor, siendo mayores puntuaciones de color sensorial cuando la adición de alfalfa es menor. A partir de ello los ensayos 1, 2 y 7 son las mejores, que son contrastados con los resultados de las Tablas 10, 11 y Figura 5.

Aroma: El maracuyá y el extracto no tienen influencia significativa. El $R^{2}(63,77)$ es bajo. No se presentan términos significativos, por lo que a partir de las medias podemos establecer que los ensayos 4,7 y 10 son las mejores.

Sabor: El extracto de alfalfa tiene influencia significativa. Pero el $R^{2}(77,64)$ es bajo no se establece relación superficie respuesta. Los ensayos 1, 2 y 7 son las mejores.

Consistencia: El extracto de alfalfa tiene influencia significativa. Pero el $R^{2}(84,11)$ es bajo no se establece relación superficie respuesta. Los ensayos 1, 7 y 9 son las mejores.

Apariencia: El extracto de alfalfa tiene influencia significativa. Pero el $R^{2}$ es bajo no se establece superficie de respuesta. Las formulaciones 1 , 2 y 7 son las mejores.

\section{Intención de compra}

Se evaluó la intención de compra con 5 puntos, siendo 5 el máximo puntaje (Ciertamente compraría) y 1 el mínimo puntaje (ciertamente 
no compraría).

El extracto de alfalfa tiene influencia significativa. Pero el $R^{2}$ es bajo y no se establece superficie de respuesta.

Las formulaciones 2, 3 y 7 son las mejores.

Las propiedades físico químicas del producto final como porcentaje de sólidos solubles, $\mathrm{pH}$ y acidez, son influenciadas directamente por el zumo de maracuyá, en tanto los atributos sensoriales como color, aroma, sabor, consistencia y apariencia son relacionadas por la interacción del zumo de maracuyá y alfalfa.

La formulación más recomendable se logra a partir de la mezcla de $25,6 \%$ de zumo de maracuyá y $2,9 \%$ de extracto de alfalfa. El producto final es factible de incursionar en el mercado por las bondades nutricionales que éste representa.

\section{REFERENCIAS BIBLIOGRÁFICAS}

Franco, J., Sulca, L, Delgado, P., Cáceres, C. \& Auccasi, A. (2003). Diversidad florística medicinal altoandina y propuesta de aprovechamiento de especies endémicas como recurso terapéutico del departamento de Tacna. Universidad Nacional Jorge Basadre Grohmann, Tacna.
Gonzales, I., Jiménez, F., Pérez, A., Rangel, E., \& Serrano, T. (2003). Elaboración de una Bebida a partir de la alfalfa, saborizada con piña. Universidad Metropolitana. México. División de Ciencias Biológicas y de la Salud. Departamento de Biotecnología. 74-79.

Jamanca, N. (2013). Potencialidades agroindustriales de los cultivos en Huarmey. IESTP Huarmey.

Reyes, M., Gómez, I., Espinoza, C., Bravo, F., y Ganoza, L. (2009). Tablas peruanas de composición de alimentos peruanos. Centro Nacional de Alimentación y Nutrición - Instituto Nacional de Salud. 8va edición.

Rodríguez, M., \& Lemma, A. (2005). Planejamento de experimentos e otimização de processos. Editorial Casa de Pão. Brasil.

\section{Correo electrónico:}

njamanca@hotmail.com

Revisión de pares:

Recibido: 15-05-2017

Aceptado: 26-06-2017 